\title{
IDADE DE APARECIMENTO E DESAPARECIMENTO DAS PONTAS ROLÂNDICAS EM 160 CRIANÇAS ACOMPANHADAS AMBULATORIALMENTE
}

\author{
ESTUDO ATUARIAL
}

\author{
MOACIR A. BORGES*, MOACIR F. GODOY**, MARCELO SCARABEL ***
}

\begin{abstract}
RESUMO - Proposta: Determinar a idade em que surgem e desaparecem as pontas rolândicas (PR) em traçados eletrencefalográficos de rotina. Método: Estudo prospectivo hospitalar, baseado em 412 eletrencefalogramas de 160 crianças que frequentaram o ambulatório de neuropediatria entre as idades de 1 e 16 anos, no período de março de 1989, a março de 1998. Usou-se o sistema 10/20 para colocação dos eletrodos. As crianças foram dividas, por idade, em quatro grupos ( 1 a 4; 5 a $8 ; 9$ a 16; 1 a 16) e usou-se o método de curva atuarial, considerandose como evento, o desaparecimento da PR. Resultados: PR teve distribuição entre meninos e meninas na razão de 64/36. A idade média em que surgiu a PR foi 7 anos (7,2 para homens e 6,6 para mulheres) e, num pequeno grupo de crianças que tinha EEG normal anterior ao surgimento da PR, a idade média foi 6,8 anos. Após 7 anos de seguimento, a percentagem de pacientes livres de PRs era $60 \%$ para o grupo de 1 a 4 anos na admissão, $84 \%$ para os grupos de 5 a 8 anos e 9 a 16 anos. Para o grupo total ( 1 a 16 anos) a percentagem de livres de PR, após 7 anos de seguimento, foi $78 \%$. Conclusão: $\mathrm{O}$ estudo mostra que as $\mathrm{PR}$ surgiram, em média, aos 7 anos e têm probabilidade de desaparecimento até 7 anos de seguimento, independetemente da faixa etária de aparecimento.
\end{abstract}

PALAVRAS-CHAVE: ponta rolândica, epilepsia focal benigna da infância com ponta rolândica, curva atuarial.

\section{Age of appearence and disappearence of rolandic spikes of 160 children: an actuarial study}

ABSTRACT: This study aims to determine the age at which the rolandic spikes (RS) appear and disappear in routine EEGs. Method: It has been carried out a hospital based prospective study of 412 EEGs records of 160 children who had been assisted at the neuropediatric out-patient department during the period between March, 1989 and March,1998. Recordings were made on 8-channel instruments and 10/20 system has been to place the electrodes. The children have been divided into 4 groups, according to their age (1 to $4 ; 5$ to $8 ; 9$ to $16 ; 1$ to 16 ), and the actuarial curve method has been used to show the disappearance of the rolandic spikes. Results: RS distribution between boys and girls has been 64/36 ratio. RS have been found at average age of 7 years (boys, 7.2 and girls, 6.6). In a small group of children with normal EEGs previous to the RS appearance, the average age has been 6.8 years old. After this 7-year follow up period, the percentage of patients free from RSs was $60 \%$ for 1 to 4 year old group at admission; $84 \%$ for the 5 to 8 year old group and for the 9 to 16 year old group. For the total group (1 to 16 year old), the percentage of those free from RSs after this 7 year follow up period was 78\%.Conclusion: This study shows that RSs appear at the average age of 7 years old, and are likely to disappear up to the end of a 7-year follow up period, independently on the appearance age.

KEY WORD: rolandic spikes, centrotemporal spikes, benign childhood epilepsy with centrotemporal spike.

Serviço de Epilepsia do Departamento de Ciências Neurológicas da Faculdade de Medicina de Rio Preto (FAMERP). *Chefe de Serviço; **Coordenador da Pós Graduação da FAMERP (Área Medicina), ***Médico Residente. Aceite: 21-junho-1999.

Dr. Moacir Alves Borges - Av Faria Lima 5622 - 015090-000 São José do Rio Preto SP - Brasil. E-mail: moacirb@zaz.com.br 
A epilepsia parcial benigna da infância(EPBI-R) foi associada às pontas nas áreas rolândicas há 20 anos, por Nayrac e Beaussart ${ }^{1}$ e Bancaud et al. ${ }^{2}$. Entretanto, foi Martinus Rulandus, no século XVI, quem fez a descrição clínica desta síndrome epiléptica ${ }^{3}$. Lombroso ${ }^{4}$ denominou essas crises epilépticas de "crises silvianas" e definiu as pontas rolândicas (PRs), como sendo ondas registradas na área fronto-centro-temporo-parietal e com negatividade máxima na área centro-temporal. As PRs nem sempre estão associadas à epilepsia ${ }^{4-7}$; entretanto são elementos importantes da EPBI-R, cuja fenomenologia tem as seguintes características principais: crises parciais motoras hemifaciais breves, crises parestésicas envolvendo a língua, lábios, gengivas, face interna da bochecha, região lateral da garganta, anartria e hipersalivação ${ }^{8}$. As PRs têm caráter genético bem determinado segundo os estudos dos grupos de Bray e de Degen ${ }^{9-12}$.

A EPBI-R ${ }^{13}$ é a epilepsia mais frequente na infância, sendo encontrada entre 13 e $23 \%$ de todas crianças epilépticas $^{11,14}$ e predomina nos meninos na razão de 60:40 ${ }^{13,15}$. Após a descrição das ondas pontas na região temporal média ${ }^{16}$, Gibbs e Gibbs ${ }^{17}$, na década de cinquenta, verificaram que a normalização do eletrencefalograma (EEG) era maior nas pessoas que estavam sem crises e Blom e Heijbel ${ }^{18}$ relataram a normalização em todos os seus pacientes com PR após 10 anos de seguimento.

O objetivo deste estudo é determinar as idades nas ocasiões do EEG inicial e final com PRs.

\section{MÉTODO}

O estudo analisa prospectivamente 412 EEGs com PR diagnosticados, por ordem de chegada, em 160 crianças, que frequentavam o ambulatório de pediatria sendo $103(64,4 \%)$ do sexo masculino, na faixa etária de 1 a 16 anos entre março de 1989 e março de 1998.

Os principais motivos para a realização do primeiro EEG foram: crises epilépticas em 88 (55\%) crianças, dificuldade escolar ou comportamental em 28 (17,5\%), cefaléia em 24 (15\%) e outros motivos em 20 (12,5\%). Cem crianças $(62,5 \%)$ tinham epilepsia, $45(28,1 \%)$ não tinham e em 15 o diagnóstico de epilepsia foi incerto. Programou-se a realização de um exame anual para cada criança, porém nem sempre foi conseguido.

Os EEGs foram analisados conforme critérios de PR de Lerman ${ }^{19}$, isto é: ritmo de base normal, PR com tendência a forma dipolar com eletropositividade frontal, descargas que ocorreram em salvas, localização, ora num, ora noutro hemisfério cerebral, acentuação e tendência à generalização das descargas durante sono lento e desaparecimento até a adolescência.

Os EEGs de crianças com déficits neurológicos e deficiência mental $(\mathrm{QI}<80)$ foram excluídos.

Os exames foram realizados em aparelhos da marca Berg, Modelo T.P. 119 de 8 canais com constante de tempo de 0,3 segundos, filtro $70 \mathrm{~Hz}$, velocidade do papel $3 \mathrm{~cm} / \mathrm{s}$. A duração dos exames em vigília, incluindo a prova de hiperpnéia de 3 minutos e foto-estimulação na frequência de $14 \mathrm{~Hz}$, foi de 15 minutos. Foram realizados 56 exames em sono induzido com hidrato de cloral e 5 exames ao longo de toda noite. Foi usado o sistema 1020, segundo Jasper ${ }^{20}$, para colocação dos eletrodos.

Considerou-se como normalização do traçado, a ocorrência de, no mínimo, dois EEGs normais realizados em vigília, em intervalo mínimo de 1 ano.

Usou-se o método de curva atuarial (Kaplan-Meier), considerando-se, como evento, o desaparecimento da PR para análise dos resultados de todos EEGs na faixa etária de 1 a 4, 5a 8, 9 a 16 e 1 a 16 anos.

A comparação dos resultados com os da literatura baseou-se no intervalo de confiança $(\mathrm{Cl}, 95 \%)$ e $t$ de Student. O nível de significância usado foi $p \leq 0,05$. Utilizou-se o programa GraphPad InStat para a construção das curvas atuariais.

\section{RESULTADOS}

A idade média foi 7 (limite entre 1 e 16 anos); dos meninos foi 7,2 (limite entre 1 e 16 anos) e das meninas, 6,6 (limite entre 1 e 13 anos).

Quarenta e cinco, 28,12\% (21,16-35,08) das crianças com PR não tinham epilepsia.

Vinte e seis crianças tinham um ou mais EEGs normais precedendo o exame em que foi registrado a primeira PR e, nesse grupo, a idade média foi 6,8 anos (com limite entre 1 a 15 anos).

As PRs foram encontradas em 32 (20\%) crianças na faixa etária de 1 a 4 anos, 81(50,6\%) na faixa de 5 a 8 anos e 47(29,3\%) na faixa de 9 a 16 anos. 
Tabela 1. Percentagem *anual dos EEGs ainda com PRs entre os 4 grupos.

\begin{tabular}{cccccccccc}
\hline $\begin{array}{l}\text { Período } \\
(\text { anos })\end{array}$ & \multicolumn{2}{c}{$1-16$ anos } & \multicolumn{2}{c}{$1-4$ anos } & \multicolumn{2}{c}{$5-8$ anos } & \multicolumn{2}{c}{$9-16$ anos } \\
\hline 1 & DP** & $\%$ & DP & $\%$ & DP & $\%$ & DP \\
2 & 99.37 & 0.62 & 100 & - & 100 & - & 97.82 & - \\
3 & 75.66 & 4.54 & 89.47 & 7.04 & 71.79 & 7.20 & 67.46 & 8.53 \\
4 & 55.16 & 5.87 & 57.51 & 12.32 & 57.43 & 8.62 & 48.19 & 10.17 \\
5 & 41.85 & 6.25 & 50.32 & 12.70 & 43.07 & 9.66 & 32.12 & 10.16 \\
6 & 28.63 & 6.18 & 40.26 & 13.57 & 32.30 & 9.80 & 16.06 & 8.29 \\
7 & 22.27 & 6.23 & 40.26 & - & 16.15 & 9.44 & - & - \\
\hline
\end{tabular}

*segundo a curva atuarial; ** desvio padrão.

A Tabela 1 mostra que $100 \%$ das crianças, na faixa etária de 1 a 4 anos, permaneceram com PR em seus traçados, após o primeiro ano do diagnóstico. No segundo ano, 89,4\%; no terceiro ano, 57,5\%; no quarto, 50,3\%; no quinto, sexto e sétimo ano, 40,2\% ainda apresentaram PRs.

Entre crianças na faixa etária de 5 a 9 anos, $100 \%$ delas permaneceram com PR em seus traçados, após o primeiro ano do diagnóstico. No segundo ano, $71,7 \%$; no terceiro ano, $57,4 \%$; no quarto, $43 \%$; no quinto, 32,3\%; no sexto e sétimo, 16,1\% ainda apresentaram PRs.

Entre as crianças na faixa etária de 9 a 16 anos, 97,8\% delas ainda tiveram PR em seus traçados, após o primeiro ano em que ocorreu o diagnóstico; no segundo ano, 67,4\%; no terceiro, 48,2\%; no quarto, 32,1\%; no quinto, sexto e sétimo 16,0\% ainda apresentaram PRs.

Entre as crianças na faixa etária de 1 a 16 anos, 99,3\% delas permaneceram com PR em seus traçados, após o primeiro ano do diagnóstico. No segundo ano, 75,6\%; no terceiro ano, $55,1 \%$; no quarto, 41,8\%; no quinto, 28,6\%; no sexto e sétimo, 22,2\% ainda apresentaram PRs.

A Figura 1 representa as quatro curvas atuariais dos grupos estudados e têm comportamento de normalização do EEG com PR semelhantes estatisticamente entre si, sendo que a curva em vermelho representa a curva atuarial do grupo de crianças na faixa etária de 1 a 4 anos; azul, de 5 a 8 anos; lilás, de 8 a 16 anos e preta, de 1 a 16 anos.

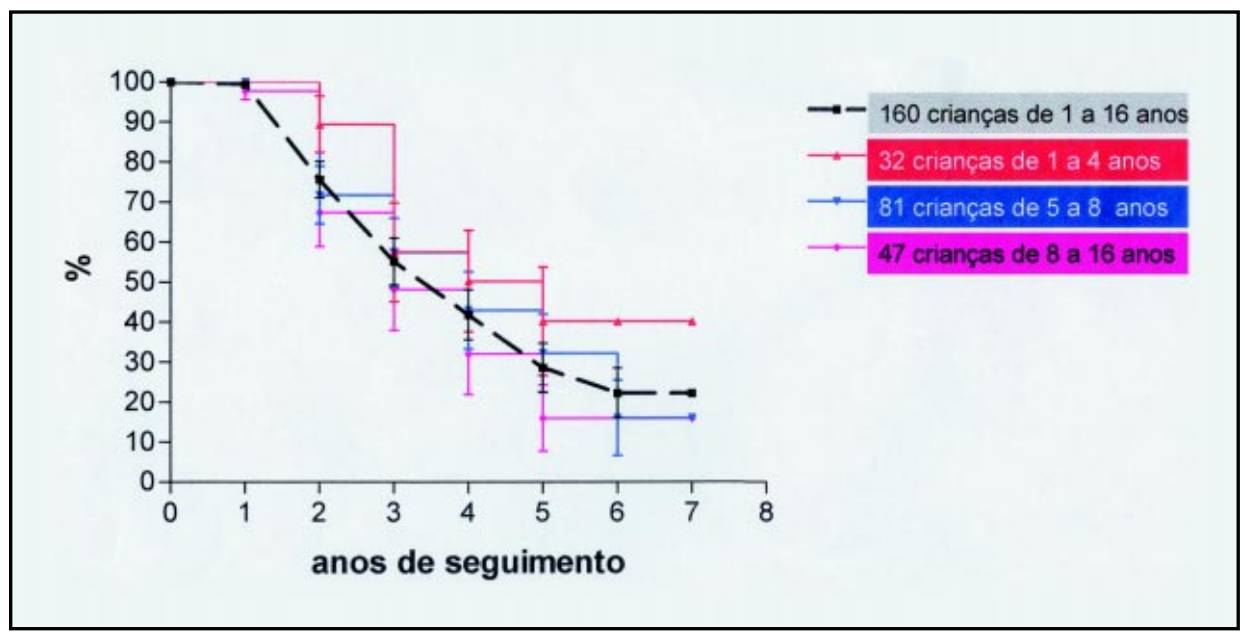

Fig 1. Curva atuarial de persistência de pontas rolândicas de acordo com as faixas etárias de diagnóstico. 


\section{DISCUSSÃO}

A idade média das crianças, quando se fez o diagnóstico de PR, foi 7 anos, mais tardiamente nos meninos (7,72 anos) que nas meninas (6,61 anos). Oito anos foi a idade média em que apareceram as PR segundo Gibbs e Gibbs ${ }^{17}$, o que foi confirmado posteriormente ${ }^{15,21}$. Por outro lado Legarda ${ }^{22}$ encontrou 6,25 anos (limite 1 e 12 anos), nas crianças com PR e epilepsia. Não há, portanto, acordo na literatura quanto à idade em que surge a PR. A idade média registrada neste estudo, por se tratar de pesquisa com base hospitalar, não pode ser considerada idade média real do aparecimento das PRs, pois não há como saber se as referidas crianças já não as teriam se o exame fosse feito em data anterior. Mesmo em pesquisa com base populacional, persistiria a dificuldade devido a esse viés. Estudo tipo "coorte", em um grupo populacional ao acaso, é o que melhor se prestaria para a determinação da idade média de forma confiável, porém com limitações impostas pelo longo tempo para acompanhamento, acrescido dos custos altos.

Considerando-se a possibilidade da idade média do surgimento da PR ser, na realidade, mais baixa $^{2,22}$, foi encontrado um grupo de $26[16,25 \%(10,53-21,96)]$ crianças em que havia, pelo menos, um EEG normal anterior ao surgimento da PR. Neste subgrupo, a idade média foi 6,85 anos (1 a 15), semelhante à idade média geral desta pesquisa e a encontrada por Legarda $(\mathrm{p}=1,09)$, porém menor que a encontrada por Lombroso $(\mathrm{p}=0,023)$. Entretanto, por se tratar de amostra pequena, deverão ser aguardados estudos conclusivos posteriores.

As quatro faixas etárias estudadas têm comportamentos semelhantes quanto ao desaparecimento das PR, pois mesmo as que mais se distanciaram entre si não mostraram diferença estatisticamente significante. Portanto', a probabilidade de normalização das PRs não depende da idade em que se diagnostica a PR nos EEGs das crianças.

Após 3 anos de evolução, foi encontrado 55,1\% de EEGs com PR. Lombroso ${ }^{4}$, ao questionar a teoria da "migração do foco temporal médio das regiões occipitais" de Gibbs ${ }^{23}$, encontrou 53,4\% de EEGs ainda com PR após 3 anos de evolução, portanto, estatisticamente semelhantes.

A atual pesquisa mostra que 28,6\% dos EEGs estudados encontravam-se com PR 5 anos após diagnóstico. Lombroso ${ }^{4}$ encontrou $25,8 \%$ se forem consideradas todas pontas temporais encontradas, portanto semelhantes estatisticamente.

Lerman ${ }^{19}$ afirmou, segundo experiência pessoal, que os EEGs com PR se normalizam precocemente dentro de 6 meses, nunca ultrapassando 6 anos após a primeira crise, fato esse não confirmado pelas curvas atuariais do presente estudo.

Há evidências na literatura ${ }^{23-25}$ de que após os 20 anos as PRs desaparecem juntamente com a epilepsia, caracterizando o excelente prognóstico. No grupo de crianças estudadas, as curvas atuariais apontam para conclusão semelhante, porém há necessidade de acompanhá-las, pelo menos, por mais 5 anos para se excluir alguns EEGs com onda ponta centro temporal lesional além de dar maior consistência ao critério de normalização com a obtenção de, pelo menos, um exame normal, em sono noturno fisiológico em cada criança.

O estudo mostra que as PRs da amostra estudada surgem em média aos 7 anos de idade e têm $78 \%$ de probabilidade de se normalizar num intervalo de tempo de 7 anos, com pico de normalização ao redor de 3 anos, independentemente da idade de aparecimento

\section{REFERÊNCIAS}

1. Nyrac $P$, Beaussart M. Les pointes-ondes prérolandiques: expression EEG très particulière. Étude électroclinique de 21 cas. Res. Neurol 1958;99:201-205.

2. Bancaud J, Colomb D, Dell MB. Les points rolandiques: un symptôme propre à l'enfant. Rev. Neurol 1958;99:206-209.

3. Hufferlen AC. A tribute to Martinus Rulandus: a $16^{\text {th }}$ Century description of benign focal epilepsy of childhood. Arc Neurol 1989;46:445-447. 
4. Lombroso CT. Sylvian seizure and midtemporal spike foci in children. Arch Neurol 1967;17:52-59.

5. Eeg-Olofson O, Petersén I, Sellden U. The development of the eletroencephalogram in normal children from the age of 1 through 15 years: paroxysmal activity. Neuropädiatrie 1971;4:375-404.

6. Mortureax Y. Étude électro-clinique de certains paroxismes d'expression rolandique chez l'enfant. Thesis. Bodeaux, 1966.

7. Loiseau P, Pestre M, Dartigues JF, Commenges D, Barberger-Gateau C, Cahadon S. Long-term prognosis in two forms of childhood epilepsy: typical absence seizure and epilepsy with rolandic (centrotemporal) EEG foci. Ann Neurol 1983;13:642-648.

8. Dalla-Bernardina B, Tassinari CA. EEG of a nocturnal seizure in a patient with "benign epilepsy of childhood with rolandic spikes". Epilepsia 1975;16:497-501.

9. Bray PF, Wiser WC. Hereditary characteristics of familiar temporal-central focal epilepsy. Pediatrics 1965;36:207-211.

10. Blom S, Heijbel J, Bergfors PG. Benign epilepsy of children with centrotemporal EEG foci: prevalence and follow-up study of 40 patients. Epilepsia 1972;13:609-619.

11. Heijbel J, Blom S Rasmuson M. Benign epilepsy of childhood with centrotemporal EEG foci: a genetic study. Epilepsia, 1975;16:285-293.

12. Degen R, Degen HE. Some genetic aspects rolandic epilepsy: waking and sleep EEGs in siblings. Epilepsia 1990;31:795-801.

13. Commission on Classification and Terminology of the International League Against Epilepsy. Proposal for classification of epilepsies and epileptic syndromes. Epilepsia 1989;28:389-399.

14. Cavazzuti GB Epidemiology of different types of epilepsy in school age children of Modena, Italy. Epilepsia 1980;21:57-62.

15. Lerman P, Kivit S. Benign focal epilepsy of childhood: a follow-up study of 100 recovered patients. Arch Neurol 1975;32:261-264.

16. Lennox WG, Gibbs EL, Gibbs EL. The inheritance of epilepsy as revealed by the eletroencephalograph. JAMA 1939;113:1002-1003.

17. Gibbs EL, Gillen HW, Gibbs PA. Disappearance and migration of epileptic foci in children. Am J Dis Child 1954;88:590-603.

18. Blom S, Heijbel J. Benign epilepsy of children with centrotemporal EEG: a follow-up study in adulthood of patients initially studied as children. Epilepsia 1982;23:629-632.

19. Lerman P. Benign childhood epilepsy with centrotemporal spikes (BECT). In Engel J Jr, Pedley TA (eds). Epilepsy: a comprehensive textbook. Philadelphia: Lippincott-Raven, 1997:2307-2314.

20. Jasper HH. Report of the committee on methods of clinical examination in electroencephalography. Electroencephagr Clin Neurophyol 1958;10:370-375.

21. Beydoun A, Garofalo EA, Drury I. Generalized spike-waves, multiple loci, and clinical course in children with EEG features of benign epilepsy of childhood with centrotemporal spikes. Epilepsia 1992;33:1091-1096.

22. Legarda S, Jayakar P, Duchowny M, Alvares L and Resnick T. Benign rolandic epilepsy: high central and low central subgroups. Epilepsia, 1994;35:1125-1129.

23. Gibbs FA, Gibbs EL. Good prognosis of midtemporal epilepsy. Epilepsia 1960;1:448.

24. Bouna PAD, Bovenkerk AC, Westerndorp RGJ and Brouwer OF. The course of benign partial epilepsy of childhood with centrotemporal spikes: Neurology 1997;48:430- 437.

25. Niedermeyer E. Epileptic seizure disorders. In Eletroencephalography: basic principles, clinical applications, and related fields. 3.Ed. Baltimore: Williams \& Wiilkins, 1993:515-517. 